\title{
Polydeoxyribonucleotide and Microlens Array-type, Nanosecond-domain Neodymium:Yttrium-aluminum-garnet Laser Treatment for Scars from Costal Cartilage Harvest Surgery: Case Series of 9 Patients
}

\author{
Keun Jae Ahn' \\ Do Yeon Kim² \\ Gwahn-Woo Cheon ${ }^{3}$ \\ Hyun Jun Park ${ }^{4}$ \\ Tae Hwan Ahn ${ }^{5}$
}

\footnotetext{
${ }^{1}$ Department of Science Education, Jeju National University, Jeju, Korea

${ }^{2} R \& D$ Center, BNV Biolab, Seoul, Korea

${ }^{3}$ Maylin Clinic (Pangyo), Seongnam, Korea

${ }^{4}$ Maylin Clinic (Apgujeong), Seoul, Korea

${ }^{5}$ Fresh Facial Aesthetic Surgery and ENT Clinic, Seoul, Korea
}

\begin{abstract}
Background and Objectives
Surgery for harvesting costal cartilage is often required for revision septorhinoplasty due to a lack of septal cartilage in patients with a severely contracted nose, and postoperative scarring on the anterolateral rib cage commonly requires additional treatment. This study aimed to evaluate the therapeutic efficacy and safety of combined polydeoxyribonucleotide (PDRN) and microlens array (MLA)-type nanoseconddomain neodymium ( $\mathrm{Nd}$ ):yttrium-aluminum-garnet (YAG) laser treatment for postoperative scars after costal cartilage harvest surgery.

\section{Materials and Methods}

Nine Korean patients with scars after costal cartilage harvest surgery treated with PDRN injections and MLA-type Nd:YAG laser treatments were retrospectively reviewed.
\end{abstract}

\section{Results}

Most of the scar lesions exhibited clinical improvement at 2 weeks after PDRN and MLA-type nanosecond-domain laser treatments, and the lesions further improved after adding more treatment sessions. The median Vancouver Scar Scale (VSS) score decreased from 6 (interquartile range [IQR]: 6-7) before combined intralesional PDRN injection and MLA-type, nanosecond-domain Nd:YAG laser treatments to 3 IIQR: 2-4) thereafter. Patient satisfaction after the combination treatments was rated as satisfactory. None of our patients reported major adverse events.

\section{Conclusion}

This case series study demonstrated that combined PDRN and MLAtype, nanosecond-domain Nd:YAG laser treatments are effective and safe for treating scars from costal cartilage harvest surgery.

\section{Key words}

Scar; Costal cartilage harvest surgery; Polydeoxyribonucleotide; Neodymium:yttrium-aluminum-garnet laser; Rhinoplasty; Septoplasty
Fresh Facial Aesthetic Surgery and ENT Clinic Kukdong Starclass Building 2F-3F, 2615

Nambusoonhwan-ro, Gangnam-gu, Seoul 06267 Korea

Tel.: +82-2-575-2033

Fax: $+82-2-575-3822$

E-mail: entahsवhanmail.net

(C) Korean Society for Laser Medicine and Surgery

(c) This is an open access article distributed under the terms of the Creative Commons Attribution NonCommercial License (http://creativecommons.org/ licenses/by-nc/4.0) which permits unrestricted noncommercial use, distribution, and reproduction in any medium, provided the original work is properly cited. 


\section{INTRODUCTION}

Regional infections and subsequent inflammatory reactions on and around nasal implants after rhinoplasty can elicit excessive fibrosis and extensive scar formation. ${ }^{1}$ These pathologic reactions can result in a severely contracted nose that may distort the nasal cartilage framework. ${ }^{1}$ Most patients require revision rhinoseptoplasty to correct the contracted nose. ${ }^{1}$ However, because patients with a severely contracted nose typically present with insufficient septal cartilage, costal cartilage harvest surgery is also required for revision septorhinoplasty. ${ }^{1-3}$ Therein, rib cartilage is usually harvested from the eighth or ninth rib and then symmetrically sculpted for a columellar strut graft depending on the size of the tip projection. ${ }^{3}$ However, postoperative scarring on the anterolateral rib cage commonly requires additional scar treatments.

Fractionated optics for neodymium (Nd):yttrium-aluminum-garnet (YAG) or alexandrite lasers have been effectively used for treating atrophic scars, hypopigmented and fibrotic scars, enlarged pores, and wrinkles. ${ }^{4,5}$ Researches have shown that an microlens-array (MLA) optic and a diffractive optical element thereof enhance wound repair by inducing the production of cytokines, chemokines, and growth factors for skin rejuvenation. ${ }^{4,5}$ Laser energy, which is delivered through fractionated optics, generates fractionated high-fluenced areas in the epidermis and upper dermis with surrounding low-fluenced areas. $^{4-7}$ In these treated areas, histopathologic features of thermally-initiated laser-induced optical breakdown (TI-LIOB) with microscopic vacuoles and pseudo-cystic cavities of various sizes are visible. - $^{4-8}$

In this report, we retrospectively reviewed 9 patients with postoperative scars after costal cartilage harvest surgery for revision septorhinoplasty. Most of the scars clinically presented with eosinophilic, atrophic, and fibrotic features with various degrees of dyschromia. We treated the scars with combined polydeoxyribonucleotide (PDRN) and MLA-type 1,064-nm nanosecond-domain Nd:YAG laser energy. The therapeutic efficacy and safety thereof in the treatment of postoperative scars after costal cartilage harvest surgery were evaluated.

\section{MATERIALS AND METHODS}

\section{Patients}

In this case series study, nine Korean patients $(3$ males and 6 females; median age: 33 years; interquartile range [IQR]: 31-47; age range: 26-61 years) with scars after costal cartilage harvest surgery were retrospectively re- viewed. None of the patients reported pertinent medical or family history. All patients underwent costal cartilage harvest surgery during revision septorhinoplasty because they exhibited insufficient septal cartilage. All nine patients had no treatment history for scars after costal cartilage harvest surgery, including systemic or topical retinoid therapy, surgical scar revision, fractional ablative or non-ablative laser treatment, microneedle radiofrequency treatment, thread implantation, or injection therapies with fillers or tissue activators, except for a pulsed dye laser. This study was followed by the Institutional Review Board's regulation.

\section{Costal cartilage harvest surgery}

In each patient, cartilage from the eighth rib was harvested for a columellar strut graft after making a 3-cmwidth skin incision on the right anterolateral chest wall under general anesthesia. Then, the periosteum, muscle, and fascia layers were closed with 3-0 synthetic absorbable coated polyglactin 910 sutures (Coated Vicryl ${ }^{\mathrm{TM}}$; Ethicon Inc., Somerville, NJ, USA), and the subcutaneous fat layer was closed with 4-0 synthetic absorbable coated polyglactin 910 sutures (Coated Vicryl ${ }^{\mathrm{TM}}$; Ethicon Inc.). The skin was closed using a subcuticular suture technique with 5-0 synthetic absorbable monofilament poliglecaprone 25 sutures (Monocryl ${ }^{\top M}$; Ethicon Inc.). The postoperative wound dressing was performed using a soft silicone adhesive Mepilex ${ }^{\circledR}$ Border Post-Op (Mölnlycke Health Care, Gothenburg, Sweden). The sutures were removed after 7 days, and the incision wounds were followed up for 14 days thereafter. Then, 585-nm pulsed dye laser (Cynergy $^{\mathrm{T} M}$; Cynosure, Westford, MA, USAl treatments were performed on the postoperative scar lesions in 8 patients (88.9\%) at 1-month intervals (median number of sessions: 1; IQR: 1-2; range 0-2). The treatment parameters thereof included a pulse duration of 10-20 ms, a fluence of 8-10 $\mathrm{J} / \mathrm{cm}^{2}$, a spot size of $7 \mathrm{~mm}$, and one pass. However, the results were unsatisfactory, and the scar lesions still exhibited eosinophilic, atrophic, and fibrotic features with varying degrees of dyschromia.

\section{Postoperative scar treatment using PDRN and an MLA-type Nd:YAG laser}

After obtaining written informed consent, the patients were treated with a median of two sessions (IQR: 1-3; range 1-5) of intralesional PDRN (Placentex ${ }^{\circledR}$; PharmaResearch, Seongnam, Koreal injection and 1,064-nm, MLA-type, nanosecond-domain Nd:YAG laser treatment (Miin ${ }^{\text {TM }}$ laser; LTRA Global Co., Ltd., Seongnam, Korea) at 1 -month intervals. The skin along the linear scar was first 
cleansed with 70\% ethanol, and EMLA cream was applied under occlusion for 1 hour. Then, PDRN was injected into the scar at three points at a total amount of $0.3 \mathrm{~mL}$ using a 31-gauge, 1-mL insulin syringe, and afterwards, three non-stacking passes of MLA-type, nanosecond-domain $\mathrm{Nd}$ :YAG laser treatment were delivered. The treatment parameters thereof include a laser energy of $700 \mathrm{~mJ}$; a spot size of $10 \mathrm{~mm}$ with the MLA-type handpiece; $140 \mathrm{mi}$ crobeams/spot,; a microbeam energy of $5 \mathrm{~mJ}$; a fluence of $1.79 \mathrm{~J} / \mathrm{cm}^{2}$; a frequency of $5 \mathrm{~Hz}$; and a distance setting between the microlens and the skin surface of $40 \mathrm{~mm}$ (Fig. 1). Neither a dynamic cooling device nor air cooling device was used during the treatments. Immediately after combination treatment, the treated scar lesions were cooled with icepacks, and a Mepilex ${ }^{\circledR}$ Border Post-Op
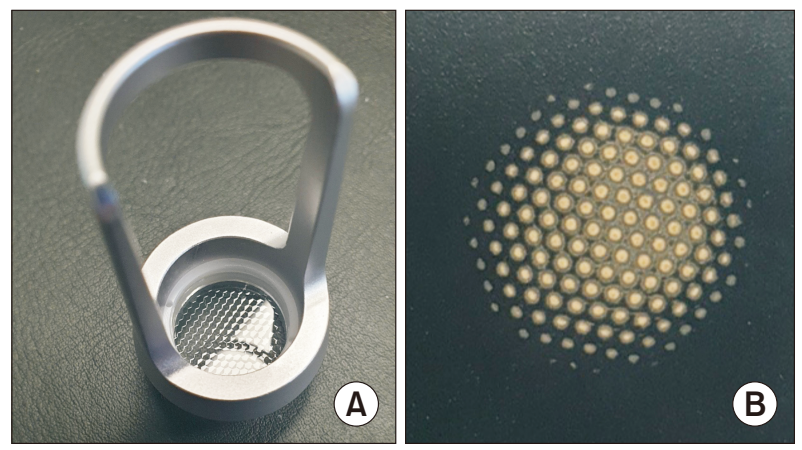

Fig. 1. Two-dimensional characterization of the microlens array (MLA)-type beam. (A) A photograph of the MLA-type handpiece. (B) Two-dimensional characterization of microbeams was evaluated by delivering MLA-type beams of the nanosecond laser on photosensitized paper (ZAP-IT ${ }^{\circledR}$; Zap-It Laser, Concord, NH, USA) at a laser energy of $700 \mathrm{~mJ}$; a spot size for the MLA-type handpiece of $10 \mathrm{~mm}$; 140 microbeams/spot; microbeam energy of $5 \mathrm{~mJ}$; a fluence of $1.79 \mathrm{~J} / \mathrm{cm}^{2}$; and the distance setting between the microlens and the skin surface of $40 \mathrm{~mm}$. was applied for 2 days. No prophylactic systemic or topical agents, including corticosteroids and antibiotics, were used. The patients were recommended to avoid excessive scrubbing and sun exposure.

\section{Objective and subjective outcome assessment}

Photographs were obtained using identical digital camera settings, lighting conditions, and patient positioning at 14 days after suture removal and every visit before performing pulsed dye laser or combined intralesional PDRN injection and the MLA-type, nanosecond-domain Nd:YAG laser treatment. At every visit and 1 month after the final treatments, physicians graded the post-costal cartilage harvest scar status using the Vancouver scar scale (VSS), which assesses pigmentation $(0$, normal; 1, hypopigmented; 2 , mixed pigmentation; 3 , hyperpigmented), vascularity (0, normal; 1 , pink; 2 , red; 3 , purple), pliability (0, normal; 1, supple; 2 , yielding; 3 , firm; 4, banding; 5 , contracture), and height (0, flat; $1, \ltimes 2 \mathrm{~mm} ; 2,2-5 \mathrm{~mm} ; 3, \pi 5 \mathrm{~mm}$ ). The score for each parameter was assessed separately, and then all four parameters were combined. At the final visit, patients also scored their overall rates of satisfaction 10 = unsatisfied, 1 = slightly satisfied, 2 = satisfied, 3 = very satisfied). Moreover, our patients were asked to report any side effects, including pain, transient erythema, swelling, oozing, itching, petechiae, bruise, bleeding, infection, ulceration, prolonged erythema, dyspigmentation, granuloma formation, and worsening of scars, over the followup period.

\section{RESULTS}

\section{Clinical course and adverse events of PDRN and MLA-type Nd:YAG laser treatment}

Immediately after each treatment, pinpoint bleeding
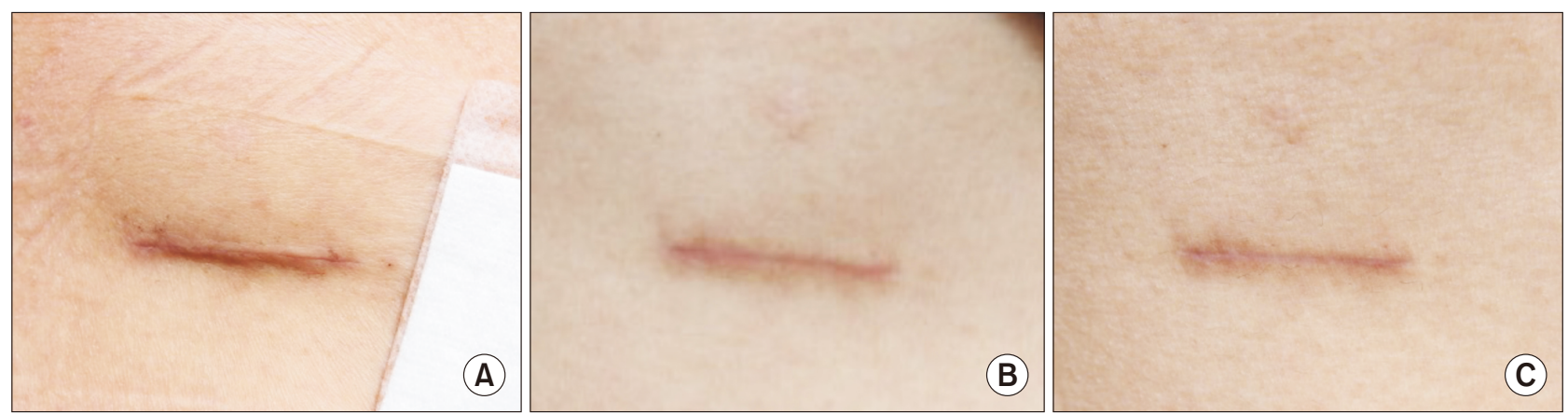

Fig. 2. Photographs of a 47-year-old Korean female patient with postoperative scarring after costal cartilage harvest surgery for revision septorhinoplasty (A) at 2 weeks after suture removal; (B) 1 month after two sessions of pulsed dye laser treatment; and (C) 1 month after one session of combined polydeoxyribonucleotide (PDRN) and MLA-type 1,064-nm nanosecond-domain neodymium (Nd):yttrium-aluminum-garnet (YAG) laser treatment. 

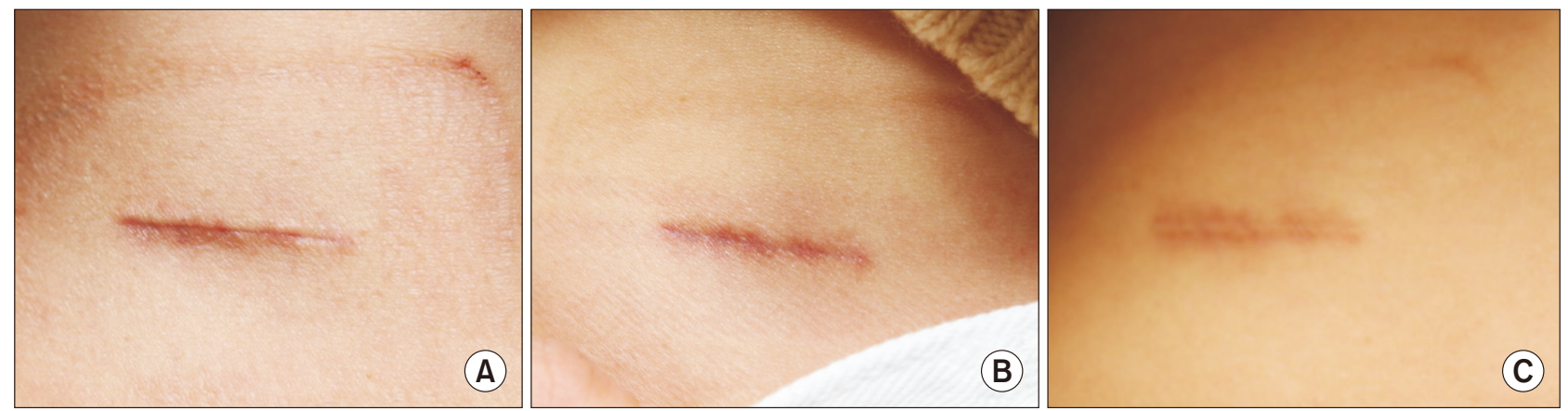

Fig. 3. Photographs of a 26-year-old Korean female patient with postoperative scarring after costal cartilage harvest surgery for revision septorhinoplasty (A) at 2 weeks after suture removal; (B) 1 month after one session of pulsed dye laser treatment; and (C) 1 month after three sessions of combined PDRN and MLA-type 1,064-nm Nd:YAG laser treatment.
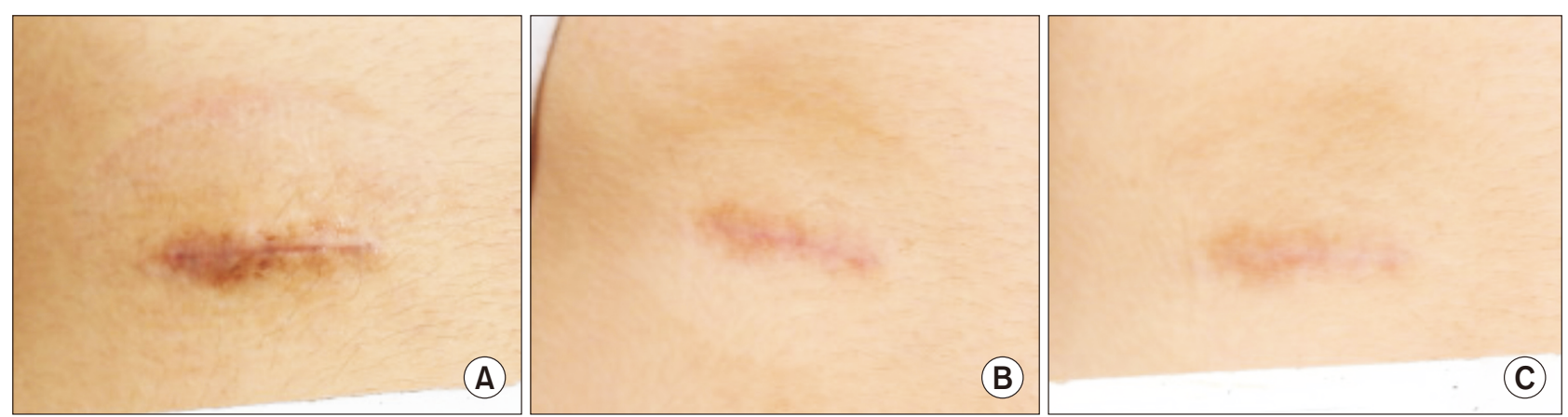

Fig. 4. Photographs of a 28-year-old Korean male patient with postoperative scarring after costal cartilage harvest surgery for revision septorhinoplasty (A) at 2 weeks after suture removal; (B) 1 month after two sessions of pulsed dye laser treatment; and (C) 1 month after two sessions of combined PDRN and MLA-type 1,064-nm Nd:YAG laser treatment.

after PDRN injections were sometimes encountered and well controlled with brief compression using sterile dry gauze. Neither mild pinpoint bleedings nor petechial patches, which could be associated with the MLA-type, nanosecond-domain Nd:YAG laser treatment, were noted in the postoperative linear scar lesions in all patients. Also, no noticeable oozing or crust formation was encountered. Mild post-treatment redness spontaneously resolved within 2 days. Most of the scar lesions exhibited clinical improvement at 2 weeks after each session of PDRN and MLA-type nanosecond-domain laser treatments, and the lesions were further improved after adding more treatment sessions (Figs. 2-4). Moreover, none of our patients reported side effects of pain during and after the treatments, swelling, oozing, itching, bruise, infection, ulceration, dyspigmentation, granuloma formation, or worsening of scars over the follow-up period.

\section{Post-treatment VSS scores and patient satisfac- tion rates}

The median values of VSS were estimated at 6 (IQR: 6-7) before combined intralesional PDRN injection and

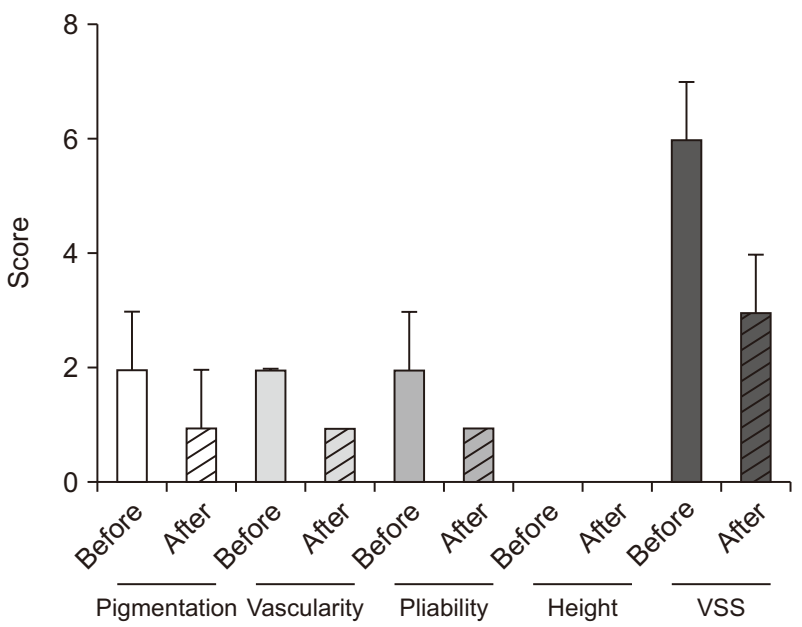

Fig. 5. The median Vancouver scar scale decreased from 6 (interquartile range [IQR]: 6-7) before combined intralesional PDRN injection and MLA-type, nanosecond-domain Nd:YAG laser treatment to 3 (IQR: 2-4) afterwards. Bars indicate interquartile range 1.

MLA-type, nanosecond-domain Nd:YAG laser treatments and at 3 (IQR: 2-4) after treatment (Fig. 5). The median values for pigmentation in VSS were estimated at 2 (IQR: 


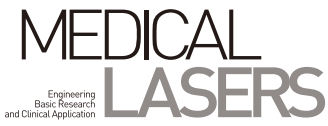

2-3) before treatment and 1 (IQR: 0-2) after treatment, while those for vascularity in VSS were estimated at 2 (IQR: 2-2) before treatment and 1 (IQR: 1-1) after treatment. Additionally, the median values for pliability in VSS were estimated at 2 (IQR: 2-3) before treatment and 1 (IQR: 1-1) after treatment, while those for height in VSS were estimated at 0 (IQR: 0-0) before treatment and 0 (IQR: 0-0) after treatment. Patient satisfaction after the combination treatment was rated as satisfactory (2, IQR: 2-3).

\section{DISCUSSION}

In the present study, we described the therapeutic efficacy and safety of combined PDRN and MLA-type, 1,064$\mathrm{nm}$ nanosecond-domain Nd:YAG laser treatments for the scars of eosinophilic, atrophic, and fibrotic features from costal cartilage harvest surgery. Until recently, laserinduced tissue reactions by fractionated Nd:YAG or alexandrite laser treatments have been investigated mainly using picosecond-domain lasers. ${ }^{9-11}$ Therein, MLA-type, picosecond-domain laser energy generates fractionated high-fluenced areas in the epidermis and upper dermis with surrounding low-fluenced areas. ${ }^{10}$ These areas have been shown to exhibit histopathologic features of microscopic vacuoles and pseudo-cystic cavities in ex vivo pigmented micropig skin. ${ }^{10}$ Moreover, the patterns of MLAtype laser-induced tissue reactions have been found to differ according to the wavelength (532 $\mathrm{nm}$ or 1,064 nm), fluence, and focusing depth (distance between the microlens and skin) of the laser energy. ${ }^{10}$ Accordingly, MLAtype Nd:YAG lasers have been suggested to be of use for various clinical purposes, including skin rejuvenation, atrophic scars, enlarged pores, and pigmentary disorders, depending on the treatment parameters. ${ }^{6-11}$

Picosecond-domain lasers have been shown to elicit notably greater photomechanical effects on target tissue than nanosecond-domain lasers. ${ }^{9.10}$ A previous ex vivo pigmented micropig study demonstrated that picosecond-domain laser irradiation results in larger and more microscopic vacuoles and pseudo-cystic cavities in the epidermis and upper dermis than nanosecond-laser irradiation at similar treatment settings, including the type of beam (flat-top beam), wavelength (532 nm and 1,064 $\mathrm{nm})$, and number of pulses. ${ }^{10}$ Moreover, multiple stacking pulses of MLA-type, picosecond-domain laser treatment has been found to generate marked TI-LIOB reactions in the epidermis and remarkably large areas of thermal coagulation in the mid to deep dermis of ex vivo brown micropig skin. ${ }^{7}$ While multiple non-stacking pulses of MLA-type laser treatment produced more homogeneous, numerous microscopic vacuoles and pseudo-cystic cavities in the epidermis and upper dermis, compared with stacking pulses, large areas of dermal coagulation could not be found?

A pattern analysis study using tissue-mimicking phantom revealed that nanosecond-domain laser treatments effectively fragmented imbedded tattoo particles into smaller ones, albeit more coarsely disintegrated, than picosecond-domain laser treatments at similar treatment settings. ${ }^{9}$ Nonetheless, nanosecond-domain laser treatments reduced the risk of photomechanical damage to the surrounding tissues, and photomechanical reactions were found to be stabilized earlier with nanoseconddomain lasers than with picosecond-domain lasers. ${ }^{9}$ We suggest that the intensification of nanosecond-domain laser-induced tissue reactions, including the formation of microscopic vacuoles and small pseudo-cystic cavities, by adapting MLA-type energy delivering technology and treatment at high energy could have resulted in the clinical improvement of the postoperative scars in our patients. ${ }^{9.10}$

In this report, we also use intralesional injection therapy with PDRN in combination with MLA-type, 1,064-nm nanosecond-domain Nd:YAG laser treatment to treat postoperative scars. PDRN is efficacious in promoting wound repair and restoration of the skin and skin appendages by stimulating neovascularization and fibroblast differentiation and maturation. ${ }^{12-14}$ Previously, our study group reported that nasal skin in patients with a severely contracted nose was markedly improved by performing repetitive PDRN injections and invasive pulsed radiofrequency treatments before and after revision rhinoseptoplasty. ${ }^{2}$ In this study, we suggested that the intralesional injection of PDRN could enhance the wound repair and remodeling of microscopic treatment zones generated by delivering MLA-type, nanosecond-domain Nd:YAG laser pulses to scars from costal harvest surgery.

There are a few limitations to this study that warrant consideration. This case series study reviewed only a small number of patients. Also, the individual efficacies of PDRN and MLA-type, nanosecond-domain Nd:YAG laser treatments for treating postoperative scars were not investigated. Finally, this study lacked a placebo-controlled, split-lesion design. Therefore, further preclinical experiments for evaluating precise tissue reactions by MLAtype, nanosecond-domain Nd:YAG laser treatment and controlled in vivo investigations should be followed to confirm our findings.

In conclusion, our study demonstrated that combined PDRN and MLA-type, nanosecond-domain Nd:YAG laser 
treatments are effective and safe for treating scars from costal cartilage harvest surgery on the lower chest wall.

\section{CONFLICT OF INTEREST}

No potential conflict of interest relevant to this article was reported.

\section{FUNDING}

This work was supported by research funding from the Korean Association for Laser, Dermatology and Trichology (KALDAT-20210001). This research was also supported by the 2020 Scientific Promotion Program funded by Jeju National University. We would also like to thank Anthony Thomas Milliken, ELS, at Editing Synthase (https://editingsynthase.coml for his help with the editing of this manuscript.

\section{REFERENCES}

1. Oh YH, Seo JW, Oh SJ, Oum IT, Kim G, Kim J, et al. Correction of severely contracted nose. Plast Reconstr Surg 2016;138:57182.

2. Ahn TH, Cho SB. Adjuvant therapy for revision rhinoplasty of contracted nose using polydeoxyribonucleotide and invasive bipolar radiofrequency. Plast Reconstr Surg Glob Open 2018;6:e1645.

3. Ahn TH, Lee W, Kim HM, Cho SB, Yang EJ. Use of autologous adipose-derived stromal vascular fractions in revision rhinoplasty for severe contractures in Asian patients. Plast Reconstr Surg 2021;147:401e-11e.

4. Bernstein EF, Schomacker KT, Basilavecchio LD, Plugis JM, Bhawalkar JD. Treatment of acne scarring with a novel fractionated, dual-wavelength, picosecond-domain laser incorporating a novel holographic beam-splitter. Lasers Surg Med 2017:49:796-802.

5. Varghese B, Bonito V, Jurna M, Palero J, Verhagen MH. Influence of absorption induced thermal initiation pathway on irradiance threshold for laser induced breakdown. Biomed Opt Express 2015;6:1234-40.

6. Suh SB, Ahn KJ, Chung HJ, Suh JY, Cho SB. Human fibroblast- derived multi-peptide factors and the use of energy-delivering devices in Asian patients. Med Lasers 2020;9:12-24.

7. Lyu H, Park J, Lee HC, Lee SJ, Kim YK, Cho SB. In vivo and ex vivo skin reactions after multiple pulses of 1,064-nm, microlens array-type, picosecond laser treatment. Med Lasers 2020;9:142-9.

8. Agarwal M, Velaskar S. Laser skin rejuvenation with fractional 1064 Q-switched Nd:YAG in 252 patients: an Indian experience. J Cosmet Dermatol 2020;19:382-7.

9. Ahn KJ, Zheng Z, Kwon TR, Kim BJ, Lee HS, Cho SB. Pattern analysis of laser-tattoo interactions for picosecond- and nanosecond-domain 1,064-nm neodymium-doped yttriumaluminum-garnet lasers in tissue-mimicking phantom. Sci Rep 2017;7:1533.

10. Lee HC, Childs J, Chung HJ, Park J, Hong J, Cho SB. Pattern analysis of 532- and 1,064-nm picosecond-domain laserinduced immediate tissue reactions in ex vivo pigmented micropig skin. Sci Rep 2019;9:4186.

11. Chung HJ, Lee HC, Park J, Childs J, Hong J, Kim H, et al. Pattern analysis of 532- and 1064-nm microlens array-type, picosecond-domain laser-induced tissue reactions in ex vivo human skin. Lasers Med Sci 2019;34:1207-15.

12. Chung KI, Kim HK, Kim WS, Bae TH. The effects of polydeoxyribonucleotide on the survival of random pattern skin flaps in rats. Arch Plast Surg 2013;40:181-6.

13. Galeano M, Bitto A, Altavilla D, Minutoli L, Polito F, Calò M, et al. Polydeoxyribonucleotide stimulates angiogenesis and wound healing in the genetically diabetic mouse. Wound Repair Regen 2008;16:208-17.

14. Lee SH, Zheng Z, Kang JS, Kim DY, Oh SH, Cho SB. Therapeutic efficacy of autologous platelet-rich plasma and polydeoxyribonucleotide on female pattern hair loss. Wound Repair Regen 2015;23:30-6.

How to cite this article: Ahn KJ, Kim DY, Cheon GW, Park HJ, Ahn TH. Polydeoxyribonucleotide and microlens arraytype, nanosecond-domain neodymium:yttrium-aluminumgarnet laser treatment for scars from costal cartilage harvest surgery: case series of 9 patients. Med Lasers 2021;10:90-95. https://doi.org/10.25289/ML.2021.10.2.90 